\title{
Modelos didáticos no ensino de Botânica
}

\author{
Didactic models in Botany teaching \\ Modelos didácticos para la enseñanza de Botânica
}

Recebido: 01/04/2021 | Revisado: 09/04/2021 | Aceito: 15/04/2021 | Publicado: 28/04/2021

\author{
Ilgmir Renan de Souza \\ ORCID: https://orcid.org/0000-0001-9201-9582 \\ Secretaria Municipal da Educação: Palhano, Ceará, Brasil \\ E-mail: ilgmir.bio@gmail.com \\ Nilda Masciel Neiva Gonçalves \\ ORCID: https://orcid.org/0000-0003-1327-0803 \\ Universidade Federal do Piauí, Brasil \\ E-mail: nildabio@ufpi.edu.br \\ Ana Carolina Landim Pacheco \\ ORCID: https://orcid.org/0000-0001-6128-6485 \\ Universidade Federal do Piauí, Brasil \\ E-mail: carolandim@ufpi.edu.br \\ Maria Carolina de Abreu \\ ORCID: https://orcid.org/0000-0001-8206-7273 \\ Universidade Federal do Piauí, Brasil \\ E-mail: mariacarolinabreu@ufpi.edu.br
}

\begin{abstract}
Resumo
No Brasil, a utilização de modelos didáticos educacionais, se deu entre as décadas de 1950 e 1980, assim promovendo o ensino de forma mais efetivo. Considerando que estes mostram-se essenciais para compreensão de conteúdos de Botânica, o presente trabalho apresenta a utilização de modelos didáticos no ensino de Botânica a partir da análise de publicações das bases de dados da plataforma Periódicos CAPES, SCIELO e dos anais do Congresso Nacional de Botânica-CNBot e do Congresso Nacional de Educação-CONEDU, no período de 2005 a 2019. Especificamente, busca identificar a eficácia da utilização dos modelos didáticos; modalidades de ensino em que são utilizados; materiais para sua confecção e metodologias utilizadas com modelo didático de Botânica. A revisão mostrou que 32 publicações atenderam aos critérios de inclusão. Os trabalhos analisados apresentam aspectos positivos quanto ao uso dos modelos didáticos para o ensino de botânica, por subsidiarem a compreensão dos conceitos teóricos e comparação com os estudos morfológicos dos vegetais. Sua utilização se faz em maior escala no Ensino Superior e no Ensino Fundamental. O biscuit é o material recomendado para sua produção por sua durabilidade e preço acessível, mas outros materiais também podem ser utilizados. Os modelos didáticos são utilizados durante aulas expositivas, aulas práticas, aulas interativas, exposições, intervenções e auxiliam na aprendizagem de alunos com deficiência visual.
\end{abstract}

Palavras-chave: Recursos de ensino; Aprendizagem; Botânica.

\begin{abstract}
In Brazil, educational didactic models use occurred between the 1950s and 1980s, promoting teaching more effectively. Considering that didactic models are essential for understanding Botany contents, this work presents the didactic models use in the teaching of Botany from publications analysis in CAPES, SCIELO databases and in Annals of Congress platform National Botany-CNBot and National Education Congress-CONEDU, from 2005 to 2019. Specifically, it seeks to identify the effectiveness of using didactic models; teaching modalities in which they are used; materials for its production and methodologies used with Botany didactic model. The review showed that 32 publications met the inclusion criteria. The analyzed works present positive aspects regarding the didactic models use for botany teaching, as they support the understanding of theoretical concepts and comparison with the morphological studies of plants. Its use is made on a larger scale in Higher Education and Elementary Education. Biscuit is the recommended material for its production due to its durability and affordable price, but other materials can also be used. Didactic models are used during expository classes, practical classes, interactive classes, exhibitions, interventions and assist in the learning of visually impaired students.
\end{abstract}

Keywords: Teaching resources; Learning; Botany.

\section{Resumen}

En Brasil, la usanza de modelos didácticos pedagógicos, se incorporó en principios de 1950 y mediados de los años 1980, con la propuesta de promover una enseñanza más efectiva. Considerando que los modelos didácticos son fundamentales para el conocimiento de los contenidos de Botánica, el presente trabajo presenta el uso de modelos didácticos en clases de Botánica con análisis de publicaciones hecha en bases de datos brasileños incluyendo CAPES, 
SCIELO y anales del Congreso Nacional de Botánica (CNBot) y Congreso Nacional de Educación (CONEDU), en periodo de 2005 a 2019. En especial, este estudio busca identificar la efectividad del uso de modelos didácticos, tipos de enseñanza en las que se utilizan, materiales para su confección y metodologías empleadas con ejemplares comprensibles de Botánica. Se trata de una revisión bibliográfica en la que 32 artículos contemplaron los criterios de inclusión: estudios con intervenciones mediante el uso de modelos didácticos para la enseñanza de Botánica; estudios con detalles de la metodología para su elaboración, estudios desde 2005 hasta 2018, y artículos y anales en la lengua portuguesa. Los trabajos examinados presentan aspectos positivos en cuanto al uso de modelos pedagógicos para la enseñanza de botánica, apoyando la comprensión de conocimientos teóricos y la comparación con parámetros morfológicos de plantas. Su uso se pasa en escala más grande en la Educación Primaria y se ha propagado a la Universidad. El biscuit es el mejor material para la elaboración gracias a su durabilidad y precio accesible, pese pueden utilizar otros materiales. Los modelos son empleados durante las clases, exposiciones, clases prácticas e interactivas, exposiciones, intervenciones y además concurren en el aprendizaje de estudiantes con discapacidad visual.

Palabras clave: Herramientas de enseñanza; Aprendizaje; Botánica.

\section{Introdução}

O ensino de ciências naturais é inserido na educação básica, com competências que permitem ao aluno compreender o mundo em que vive, juntamente com os seres nele existentes. Segundo a Base Nacional Comum Curricular (BNCC) do Ensino Fundamental, apresenta o componente curricular de Ciências organizado em três unidades temáticas que se repetem ao longo do Ensino Fundamental e Médio. Essas Unidades temáticas visam levar os estudantes a, continuamente, revisitar os saberes adquiridos durante o processo de educação formal: Matéria e Energia; Vida e Evolução, Terra e Universo.

A unidade temática Vida e evolução compreende uma significativa parte do conteúdo biológico explorado no ensino básico. As plantas por serem responsáveis pelo processo de fotossíntese, resultado de seu metabolismo energético, disponibilizam para o meio: oxigênio, alimentos, matéria prima para produção de roupas, casas, fármacos, dentre outros materiais e produtos é um dos assuntos mais abordados nesse eixo temático.

Apesar da sua importância, da variedade de temas que envolvem o estudo da Botânica, o interesse para tal área no Ensino Básico não são notórios como temas de Zoologia e Genética, sendo muitas vezes considerada uma disciplina chata. O fato dos alunos não gostarem dos conteúdos de Botânica, muitas vezes se dá devido à sua complexidade teórica com vários termos técnicos que dificultam sua assimilação (Silva et al., 2017).

No ensino de ciências, muitas vezes são utilizados processos metodológicos tradicionais que acabam distanciando os alunos do objetivo da aprendizagem. Frente a esse cenário, as diretrizes apresentadas pelos Parâmetros Curriculares NacionaisPCNs orientam à seleção de estratégias que possam promover um aprendizado significativo (Brasil, 1998), possibilitando que os professores possam diferenciar suas aulas de maneira que motivem os alunos para novas aprendizagens.

Visando facilitar o processo de ensino aprendizagem, alguns professores propõem a utilização de recursos de ensino diversos, dentre eles, modelos didático-pedagógicos (Nariane et al., 2010). Entende-se por modelo didático, réplicas de modelos morfológicos, que tem por finalidade representar conceitos científicos onde o professor possa utilizar como ferramenta para expor uma determinada estrutura ou eventos biológicos, favorecendo o entendimento de fenômenos complexos e abstratos, tornando assim, o aprendizado mais concreto (Dantas et. al. 2016).

Segundo Rothe (2014), com os entraves burocráticos, éticos e financeiros que envolvem a prática de ensino, faz-se necessário estudar, avaliar e aplicar metodologias alternativas que possam substituir de forma satisfatória as aulas práticas que necessitam de laboratórios com equipamentos e materiais especializados, sendo uma realidade não existente em diversas escolas da rede pública de ensino. Para tal problema, nas aulas de Ciências, optar por metodologias alternativas que utilizam modelos didáticos apresenta-se como a melhor alternativa, considerando que, para a realização de aulas práticas, a obtenção de materiais botânicos frescos é de difícil acesso, devido a uma sazonalidade peculiar de cada região que afeta a disponibilidade de todos os órgãos das plantas para o provimento de aulas práticas (Silva et.al., 2017). 
Entretanto o uso dessas metodologias apresenta-se não somente como alternativa para aulas práticas, a mesma possui uma importância mais ampla no que tange o protagonismo do aprendizado (Silva, 2016). Dentre as diversas alternativas para o melhor aprendizado em Botânica, o uso de modelos didáticos vem sendo a melhor alternativa por ajudar o professor a estimular o raciocínio dos alunos e gerar novas aprendizagens, passando a ser o ensino mais eficaz e interessante (Almeida, 1981).

Sendo sabedores da falta de recurso didáticos no ensino público e da ausência de espaços especializados (laboratórios) para a realização de aulas práticas, alguns pesquisadores da área do ensino de Ciências têm desenvolvido modelos didáticos alternativos como forma de possibilitar aos professores instrumentos auxiliares da prática pedagógica. Também, tem sido demonstrado que a partir da utilização de materiais de baixo custo, é possível oferecer aulas mais atraentes e motivadoras, nas quais os alunos são envolvidos na construção de seu próprio conhecimento (Sousa et.al. 2008).

A utilização de um modelo didático busca não só substituir o uso de órgãos vegetais naturais nas aulas práticas como também tem como objetivo enriquecer as aulas e torná-las mais atrativas aos alunos. Também visa despertar o interesse quanto ao campo de pesquisa da Botânica, correlata à ecologia, relação às adaptações evolutivas e estruturas morfológicas e o ambiente em que determinada espécie habita.

Diante do exposto, o presente trabalho propõe uma revisão de literatura que objetiva discorrer sobre a utilização de modelos didáticos no ensino de Botânica a partir de publicações das bases de dados do Periódicos CAPES, SCIELO e dos anais do Congresso Nacional de Botânica-CNBot e do Congresso Nacional de Educação-CONEDU. Especificamente, identificar a eficácia da utilização dos modelos didáticos; modalidades de ensino em que são utilizados os modelos didáticos; materiais para confecção dos modelos didáticos e metodologias utilizadas com o uso do modelo didático de Botânica.

\section{Metodologia}

A revisão de literatura foi realizada a partir de trabalhos publicados nas bases de dados Periódicos CAPES, SCIELO, anais do Congresso Nacional de Botânica-CNBot e Congresso Nacional de Educação-CONEDU, utilizando os termos/descritores na língua portuguesa: modelos didáticos, ensino de Ciências, ensino de Botânica. No rastreamento das publicações foram utilizados os operadores lógicos "AND" e "OR", de modo a combinar os termos/descritores acima citados.

Foram utilizados como critérios de inclusão estudos que: realizaram intervenções por meio do uso de modelos didáticos no ensino de Botânica; relataram a metodologia de confecção de modelos didáticos para o ensino de Botânica, foram publicados em língua portuguesa no período de 2005 a 2019.

A seleção dos trabalhos publicados nas bases de dados Periódicos CAPES e SCIELO, ocorreu conforme Pereira (2012), partindo da leitura dos títulos, seguida da leitura dos resumos com títulos pertinentes e posterior leitura na integra dos artigos que atendem aos critérios da busca. Dos 600 artigos obtidos na busca inicial das bases de dados Periódicos CAPES e SCIELO, 58 deles foram selecionados após a leitura dos títulos ( $1^{\mathrm{a}}$ etapa) e, dentre estes, 49 foram excluídos após a leitura dos resumos e do texto na íntegra ( $2^{\mathrm{a}} \mathrm{e} 3^{\mathrm{a}}$ etapa), já que não atendiam integralmente aos critérios de inclusão.

\section{Resultados}

Para essa revisão bibliográfica 32 trabalhos foram selecionados, a saber: 09 na base de dados do SCIELO e CAPES (Tabela 1), 20 foram encontrados na base de dados do CNBot (Tabela 2), 03 na base de dados do CONEDU (Tabela 3). 
Tabela 1. Artigos aprovados após a leitura na integra de acordo com os critérios de inclusão dos trabalhos obtidos (SciELO, Google Acadêmico e WebCapes).

\begin{tabular}{|c|c|c|c|}
\hline Autor & Titulo do trabalho & Revista & $\begin{array}{c}\text { Ano de } \\
\text { publicação }\end{array}$ \\
\hline Gregório Ceccantini & Os tecidos vegetais têm três dimensões. & Revista Brasil. Bot, V.29, n.2, p.335-337 & 2006 \\
\hline $\begin{array}{l}\text { Ribeiro, J. M. M. \& } \\
\text { Carvalho, M. A. S. }\end{array}$ & $\begin{array}{l}\text { Utilização de modelos didáticos no ensino } \\
\text { de Botânica e suas implicações no processo } \\
\text { de ensino e aprendizagem. }\end{array}$ & $\begin{array}{l}\text { Revista Sapiciência: Sociedade, Saberes } \\
\text { e Práticas Educacionais, V.6, n.1, p.17- } \\
37\end{array}$ & 2017 \\
\hline Leme, F. M., et al. & $\begin{array}{l}\text { Ensino de Botânica e Educação Ambiental: } \\
\text { modelos didáticos e oficinas pedagógicas. }\end{array}$ & $\begin{array}{l}\text { Revista Educação Ambiental em Ação, } \\
\text { n.53, ano XIV }\end{array}$ & 2015 \\
\hline $\begin{array}{l}\text { Biagolini, C. H. \& } \\
\text { Piacitelli, L. P. }\end{array}$ & $\begin{array}{l}\text { Relato de experiência: o uso da argila na } \\
\text { produção de modelos de fitofósseis e seu } \\
\text { papel nos processos de ensino e } \\
\text { aprendizagem em PaleoBotânica. }\end{array}$ & $\begin{array}{l}\text { EBR - Educação Básica Revista, v.2, } \\
\text { n.2, p. 141-150 }\end{array}$ & 2016 \\
\hline $\begin{array}{l}\text { Silva, A. A., Filha, R. T. S. } \\
\text { \& Freitas, S. R. S. }\end{array}$ & $\begin{array}{l}\text { Utilização de modelos didáticos como } \\
\text { metodologia complementar ao ensino da } \\
\text { anatomia celular. }\end{array}$ & Biota Amazônia, v. 6, n.3, p.17-21 & 2016 \\
\hline Perini, M. \& Rossini, J. & $\begin{array}{l}\text { Aplicação de modelos didáticos no ensino } \\
\text { de biologia floral. }\end{array}$ & $\begin{array}{l}\text { International Scientific Journal, } \\
\mathrm{N}^{\mathrm{o}} 3 \text {, volume } 13 \text {. }\end{array}$ & 2018 \\
\hline $\begin{array}{l}\text { Almeida, D. M.; Pscheidt, } \\
\text { A. C. \& Coelho, C. B. }\end{array}$ & $\begin{array}{l}\text { Inovação em ensino de biologia: o } \\
\text { desenvolvimento de uma sequência didática } \\
\text { de ensino por investigação utilizando } \\
\text { modelos sintéticos de vegetais para as aulas } \\
\text { de botânica. }\end{array}$ & Inovae, São Paulo, Vol.7, pág.79-93 & 2019 \\
\hline $\begin{array}{l}\text { Fontes, G. S.; Elias, L. \& } \\
\text { Aoyama, E. M. }\end{array}$ & $\begin{array}{l}\text { Flora nativa no ensino de botânica: } \\
\text { proposta de modelo didático de fruto. }\end{array}$ & $\begin{array}{l}\text { Experiências em Ensino de Ciências } \\
\text { V.14, N.2 }\end{array}$ & 2019 \\
\hline Borges, B. T. et. al. & $\begin{array}{l}\text { Aulas práticas como estratégias para o } \\
\text { ensino de botânica no ensino fundamental. }\end{array}$ & Forscience, V.7, N.2. & 2019 \\
\hline
\end{tabular}


Tabela 2. Trabalhos referente ao uso de modelos didáticos no ensino de Botânica apresentados nos Congressos Nacionais de Botânica (CNBot) no período de 2006 a 2019, e selecionados para a pesquisa.

\section{Autor}

Rauber, I. M. F. et. al.

Batista, L. P. A. et. al.

Fontes, G. S. \& Aoyma,

E. M.

Custodio L. N.; Faria. R.

L. \& Oliveira, R. C.

Brandão, L. D. \&

Carneiro, C. E.

Pesamosca, A. M. et. al.

Lima, I. G.; Jesus, C. \& Santos

Dias, M. R. P. et. al.

Checom, R. L. A. \& Aoyama, E. M.

Sales, G. N. B. \& Lima, J. R.

Chaves, B. E. et. al.

Mendoza, A. Y.G. et. al.

Cordeiro, M. R. et. al.

Carvalho, D. C. F.; Lira, B. S, A. \& Mehlig, Ulf.

Aoyama, E. M. et. al.

Rocha, L., et al.

Junior-Silva, W. \&

Cabrini, E. C.

Souza, M. A. D. et al

Cordeiro, M. R. et. al.

Ramos, M. L. \& Lima, R. A.
Titulo dos trabalhos

A importância da utilização de modelos didáticos do ciclo do

Ascomycota para o ensino de Botânica

Modelo didático de flores para ensino de Botânica em uma escola pública de Atalaia do Norte - AM.

Modelo didático de fruto para o ensino aprendizagem de botânica

Da flor ao fruto: Um modelo didático para o ensino de

Botânica

Modelos dos ciclos de vida de briófitas e pteridófitas para portadores de deficiência visual.

Modelos didáticos: uma alternativa metodológica no ensino de botânica

O uso de Jogos e modelos com recurso para o ensino de Botânica.

Modelo Tridimensional e funcional vegetal: Praticidade $\mathrm{w}$ dinamismo no ensino de Botânica

Anatomia vegetal: Modelo didático de Botânica para o ensino médio

Edição

$57^{\circ} \mathrm{CNBot}$

$57^{\circ} \mathrm{CNBot}$

2006

$57^{\circ} \mathrm{CNBot}$

2006

$58^{\circ} \mathrm{CNBot}$

2007

$60^{\circ} \mathrm{CNBot}$

2009

$63^{\circ} \mathrm{CNBot}$

2012

$63^{\circ} \mathrm{CNBot}$

2012

$63^{\circ} \mathrm{CNBot}$

2012

$65^{\circ} \mathrm{CNBot}$

2014

$65^{\circ} \mathrm{CNBot}$

vegetal: Estudo de caso em uma turma de Agronomia.

Modelos Tridimensionais para ensino de anatomia vegetal para deficientes visuais

$67^{\circ} \mathrm{CNBot}$

2016

A construção do conhecimento de plantas carnívoras pelos

alunos do ensino fundamental no município de Benjamin

Constant-AM

Aulas práticas em anatomia vegetal para deficientes visuais:

Proposta de material didático

$68^{\circ} \mathrm{CNBot}$

Materiais didáticos para o ensino inclusivo de diversidade de plantas com sementes nos cursos de Ciências Biológicas e Naturais

Morfologia floral: modelo didático para o ensino de Botânica.

$68^{\circ} \mathrm{CNBot}$

Um modelo didático da flor para o ensino de sua morfologia e dos processos associados à das Angiospermas

$69^{\circ} \mathrm{CNBot}$

Modelos didáticos como ferramenta de ensino nas aulas de botânica no ensino superior

$69^{\circ} \mathrm{CNBot}$

Da folha carpelar ao fruto: Uso de modelos de biscuit no ensino de morfologia vegetal

$69^{\circ} \mathrm{CNBot}$

Estratégia didática para ensino inclusivo de alunos com deficiência visual no ensino de anatomia vegetal.

$70^{\circ} \mathrm{CNBot}$

$70^{\circ} \mathrm{CNBot}$ superior 
Tabela 3. Trabalhos referentes ao uso de modelos didáticos no ensino de Botânica apresentados no Congresso Nacional de Educação (CONEDU) no período de 2014 a 2019.

\begin{tabular}{lll}
\hline \multicolumn{1}{c}{ Autor } & Titulo do trabalho & $\begin{array}{c}\text { Edição } \\
\text { Ano de } \\
\text { publicação }\end{array}$ \\
\hline $\begin{array}{l}\text { Costa. P. R. A. M. et. } \\
\text { al. }\end{array}$ & $\begin{array}{l}\text { Utilização de porcelana fria na confecção de modelo didático } \\
\text { para o ensino de anatomia vegetal. }\end{array}$ & III CONEDU \\
$\begin{array}{l}\text { Marinho, B. F; Silva, } \\
\begin{array}{l}\text { A. R. V. \& Djanice } \\
\text { Oliveira, D. M. }\end{array}\end{array}$ & $\begin{array}{l}\text { Construção de um modelo didático para o ensino de botânica } \\
\text { na disciplina de práticas de ensino em biologia. }\end{array}$ & V CONEDU \\
$\begin{array}{l}\text { Silva; M. S.; Xavier, L. P } \\
\text { Arruda, E. C. P. }\end{array}$ & $\begin{array}{l}\text { Elaboração de modelo didático táctil de plantas avasculares no } \\
\text { ensino de botânica para portadores de baixa visão. }\end{array}$ & VI CONEDU \\
\hline
\end{tabular}

Fonte: Dados da Pesquisa (2018).

A revisão sistemática permitiu observar a predominância da preocupação textual quanto à eficácia de modelos didáticos para o Ensino de Botânica, seguida da preocupação com as formas de sua produção e intervenção no ensino. Isso se dá pelo fato de ser uma metodologia pouco utilizada comparada com ensino de anatomia humana e zoologia na área de ciências biológicas, o que subentende-se que os estudos iniciais devem ser, de fato, para ensinar como esses modelos são confeccionados e avaliar sua eficácia no ensino e na aprendizagem.

No Brasil, a utilização de modelos didáticos educacionais, se deu entre as décadas de 1950 e 1980, assim promovendo o ensino de forma mais efetivo, segundo Costa et. al. (2016). O uso desses modelos se mostrou viável, suprindo muitas vezes a falta de laboratórios nas escolas para a realização de aulas práticas, porém como pode ser observado na Figura 1, esses modelos são viáveis e estão sendo utilizados em diferentes níveis de ensino (do ensino fundamental ao superior).

Figura 1. Níveis de ensino que fazem uso de modelos didáticos para ensino de Botânica.

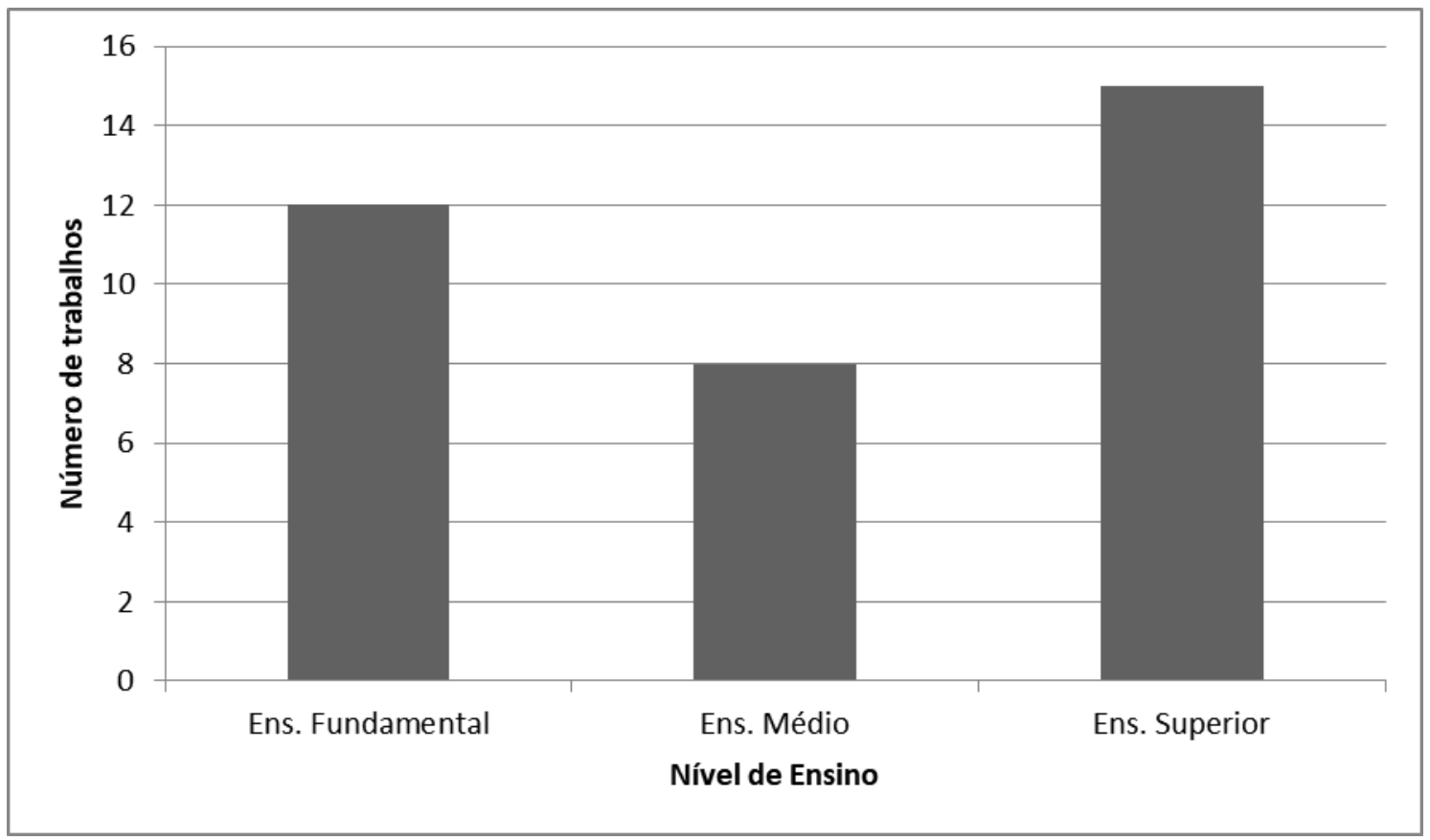

Fonte: Dados da Pesquisa (2018). 
O uso dos modelos didáticos tem se mostrado bem expressivo no ensino superior. Ceccantini (2006) relata ter implementado na disciplina de Botânica Estrutural na Universidade Federal do Paraná, modelos didáticos confeccionados por ele mesmo como parte do método de ensino e avaliação e que essa metodologia motivou os alunos em suas avaliações bem como contribuiu na formação de uma coleção de modelos de ótima qualidade. Esse trabalho serviu de inspiração para o livro “Anatoblocos: blocos didáticos para o estudo da anatomia vegetal”, publicado em 2016, por Marília Contin Ventrella. Esse material didático apresentasse extremamente útil para a compreensão da morfologia tridimensional das células. Os temas abordados são os principais tópicos da Anatomia das Espermatófitas (plantas com sementes: Gimnospermas e Angiospermas), como: células, tecidos e órgãos.

Para o ensino básico, a importância do uso de modelos didáticos, se dá pela contribuição na representação tridimensional dos órgãos e tecios vegetais, o que não é possível com a utilização do livro didático. Além de auxiliar na visualização de cortes tridimensionais, pois nas aulas práticas quando se observa cortes transversais dos órgãos vegetais, o educando tem uma visão plana da lâmina, onde muitos acabam tendo dificuldade de compreender aquela estrutura de modo especial. Dessa forma esses modelos se apresentam como ótima ferramenta para trabalhar estruturas microscópicas, visto que muitas das unidades de ensino da rede pública e privada não possuem laboratórios equipados com microscópios para aulas relacionadas à anatomia e morfologia vegetal, desse modo o uso de modelos didáticos supri essas necessidades.

A utilização desses modelos didáticos para representações de estruturas microscópicas é uma ótima alternativa metodológica para o ensino de Ciências e Biologia. Neste caso, em particular, utilizado para o ensino-aprendizagem de Anatomia Vegetal, um dos conteúdos do componente curricular teórico com práticas através do manuseio de peças que permitam um campo visual de diversos ângulos e aguça a cognição do aluno (Costa, 2016, p 4).

Para compreendermos a representação dos órgãos vegetais em modelos didáticos no ensino de Botânica, é comum que os professores abordem com mais precisão em suas aulas o grupo das Angiospermas, visto que esse grupo é o maior em número de espécies e facilmente encontrado no cotidiano dos educandos. O estudo do grupo das Angiospermas está propriamente relacionado com os seus órgãos, podendo dividi-los em dois tipos: órgãos vegetativos (raiz, caule e folha) e órgãos reprodutivos (flor, fruto e semente). $\mathrm{O}$ estudo dos órgãos vegetativos no Ensino Fundamental está diretamente relacionado com alimentação (raízes e caules) e com estudos bioquímicos (fotossíntese), já os órgãos reprodutivos estão intimamente relacionados com a reprodução/polinização (Flor) e com a alimentação (frutos e sementes).

Dentre os modelos didáticos morfológicos de órgãos vegetais mais utilizados encontram-se as flores (Figura 2), visto que esses órgãos não são facilmente encontrados devido existir um período específico de floração. As flores são órgãos reprodutivos das Angiospermas muitas vezes abordadas nas aulas de Ciências tanto pela sua morfologia (verticilos de proteção e reprodução) quanto pela formação de frutos (aspecto reprodutivo). É nas aulas de Ciências que o aluno começa a perceber que as flores que ele observa no seu dia a dia são mais que belas estruturas coloridas e perfumadas de uma planta. Os verticilos florais serão apresentados assim como suas funções.a morfologia e diversidade de estruturas entres flores diferentes e a importância desse órgão para atração de polinizadores e para reproduçao das Angiospermas. (xilema e floema), estômatos e epiderme (Raven, 2007; Nabors, 2012). Essa complexidade de estruturas e nomenclatura pouco usual faz com que se necessite de algum aparato pedagógico para melhor visualização e interpretação. 
Figura 2. Relação dos órgãos vegetais mais representados em modelos didáticos.

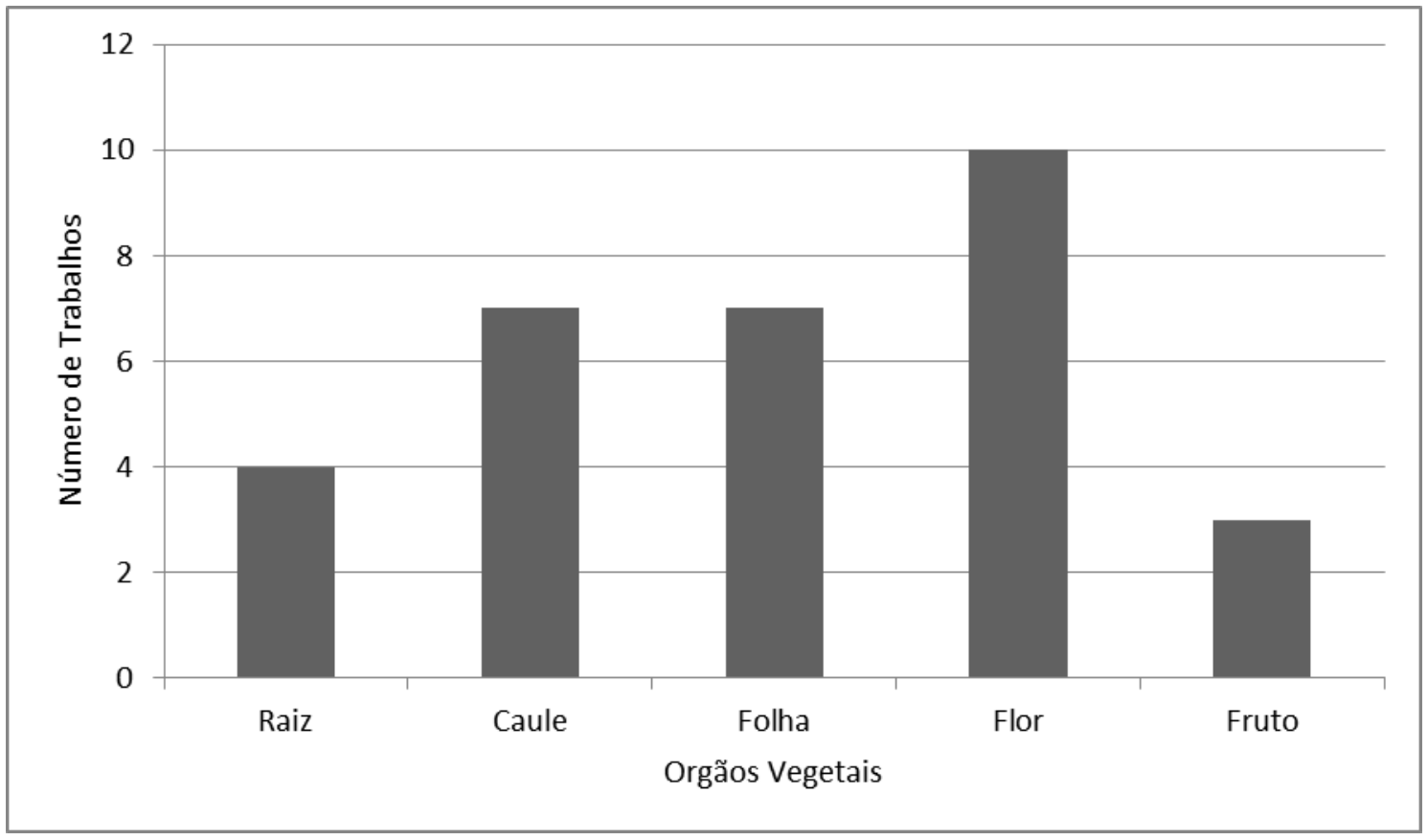

Fonte: Dados da Pesquisa (2018).

Visando o objetivo de melhor aprendizado e integração dos alunos, muitos professores têm buscado nos modelos didáticos um suporte pedagógico, complementando o uso do livro didático em suas salas de aula. Este modelo tem sido confeccionado de diversas formas, desde materiais recicláveis e de baixo custo, tais como: papelão, garrafas pet's, placas de isopor, tintas do tipo acrilex, canudinhos, miçangas, algodão e emborrachados EVA (Silva et. al. 2016; Leme et. al. 2018; Rauber et. al. 2006; Checom \& Aoyama, 2014; Fontes \& Aoyama, 2016; Brandão \& Carneiro, 2009), estes recursos tem suma importância a facilidade de obtenção e manoseio, bem como baixo cuso de aquisição.

Dentro os materiais utilizados para a confecção de modelos didáticos, o que se mostra menos eficiente é de fato a massa de modelar, pois a mesma não apresenta uma consistência firme e retém muita umidade, o que pode com o tempo proporcionar a infestação de microrganismos e fungos. Para solucionar tal problema, Ribeiro \& Carvalho (2017), sugere o uso de porcelana fria, mais conhecida como biscuit. O uso desse material se mostra realmente eficiente, pois muitos dos materiais sintéticos comercializados apresentam erros de conceito e custo muito elevado. Deste modo, o uso de modelos didáticos confeccionados com massa de biscuit (Costa et. al. 2014; Junior-Silva, Cabrini, 2018; Saraiva et. al. 2018) apresenta-se como a melhor alternativa, levando em consideração custo e benefício.

Outra técnica utilizada no ensino de botânica, no que se refere à fitofósseis, é a utilização de argila (Biagolini \& Piacitelli, 2016), pois a mesma demonstra de forma prática como que esses organismos conseguem preservar-se ou até mesmo deixar seus rastros na terra por milhões de anos. Devido à fragilidade desses materiais e por vezes muitas das amostras se tratarem de materiais científicos, a confecção de modelos didáticos destes organismos torna-se uma ferramenta necessária, nos processos de aprendizagem de forma prática e lúdica.

Visando o ensino de Botânica mais efetivo, alguns trabalhos realizados tiveram como objetivo avaliar se as metodologias aplicadas mostravam-se eficientes ou não para esse processo. Para tanto, utilizavam questionários antes e após a aplicação da metodologia para fins de comparação. Outros trabalhos tiveram como objetivo relatar a experiência em usar modelos didáticos e descrever os procedimentos de tal metodologia bem como sua viabilidade. Após a análise dos trabalhos selecionados, foram detectados 08 (oito) momentos de uso de modelos didáticos para o ensino de Botânica, sendo eles: 
I. Aulas Expositivas (AE): Nessas aulas os professores usavam modelos didáticos para apresentar imagens que estavam ilustradas no livro didático.

II. Aulas Práticas (AP): Nessas aulas os professores usavam modelos didáticos para compreensão de conteúdo abordado em sala nas práticas laboratoriais.

III. Aulas para Portadores de deficiência visual (APDV): Nessas aulas os alunos podiam apalpar os modelos didáticos.

IV. Atividade de monitoria (AM); Nessas aulas, os alunos podiam manusear os modelos didáticos e correlacionar a imagem do modelo com o conteúdo visto em sala de aula.

V. Intervenção escolar (IE): Nessas intervenções os alunos eram apresentados aos modelos didáticos e posteriormente questionados sobre eles.

VI. Exposição (EX): Na exposição era mostrada aos alunos a diversidade de morfologia dos vegetais, indagando-os e instigando-os a aprofundar seus conhecimentos.

VII. Aulas interativas (AI): Nessas aulas os alunos eram protagonistas na confecção dos modelos didáticos, o que enfatiza mais ainda os processos de ensino e de aprendizagem.

VIII. Aulas capacitivas (AC): Nessas aulas, discentes de Licenciatura em Ciências Biológicas recebiam informações sobre como aprimorar suas aulas com o uso de modelos didáticos e confeccionavam os mesmos.

Figura 3. Relação dos órgãos vegetais mais representados em modelos didáticos.

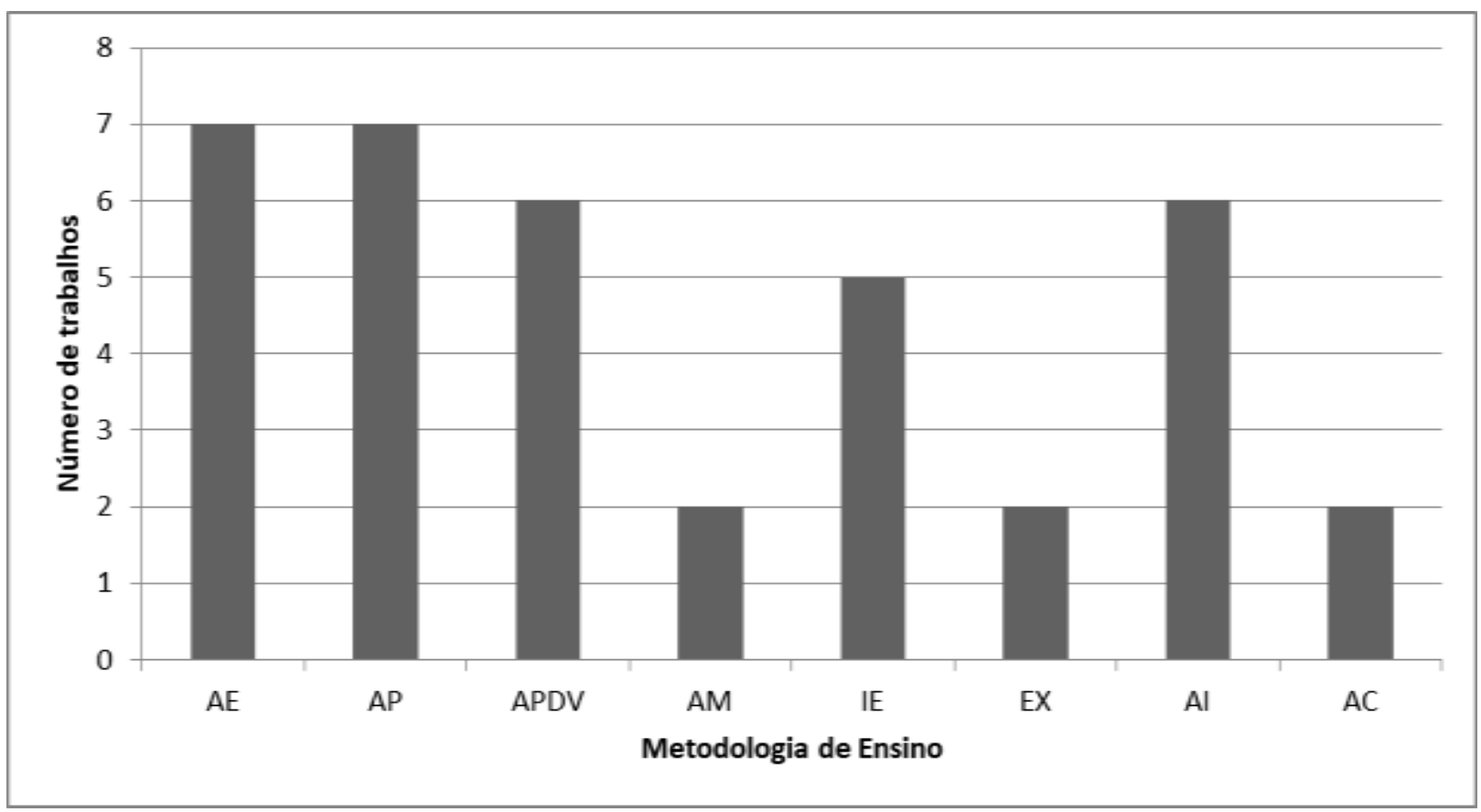

Fonte: Dados da Pesquisa (2018).

O momento oportuno para sua utilização é vasto e vai sempre de encontro aos objetivos propostos para a aula, assim, a criatividade e a motivação docente para sua inserção nos momentos da aula é essencial para a compreensão de conteúdos de Botânica.

Observa-se que os modelos didáticos para o ensino de Botânica podem ser de diversas formas, desde modelos referentes à Citologia (Junior-Silva \& Cabrini, 2018; Silva et. al, 2018) até maquetes que representam os ciclos de vida, tais como dos fungos (Rauber et. al., 2006), Briófitas e Pteridófitas (Brandão \& Carneiro, 2009) e ciclos reprodutivos das Angiospermas (Pesamosca et. al., 2012). 
Cabe ressaltar que além da observação e manuseio dos modelos didáticos, também é interessante sua produção por parte dos alunos. A produção deve partir da importância de interação aluno-matéria. Em aulas interativas, o aluno, ao confeccionar um modelo didático, terá que inicialmente realizar uma pesquisa bibliográfica sobre o determinado órgão a ser confeccionado e posteriormente elaborar suas organelas ou estruturas, desse modo o educando consegue correlacionar o estudo teórico com a prática, algo que muitas vezes não se conseguiria alcançar quando se apresenta ao educando os modelos já prontos.

Sobre as aulas para portadores de deficiência visual, partindo do princípio da inclusão onde envolve o fato de que a sociedade deve fornecer condições básicas para que todas as pessoas possam ser uma força ativa na sociedade, o processo de ensino e aprendizagem vive um desafio contínuo para a inclusão. Portanto, ao buscar a inclusão em sala de aula, os professores muitas vezes inovam seus métodos de ensino para incentivar e garantir que todos compreendam o conteúdo do trabalho em sala de aula.

Para o ensino de botânica, este tema tem se tornado muito comum nos últimos anos (Silva, 2019. Cordeiro, 2019. Carvalho, 2017. Cordeiro, 2017. Chaves, 2016. Brandão, 2009), o que mostra a atenção dos professores no planejamento de suas aulas, sendo os conteúdos trabalhados de forma mais inclusiva e promovendo a interação entre os alunos. Esses modelos de ensino também são muito importantes em termos de acessibilidade, pois a partir desses modelos o aluno com deficiência visual consegue conectar a teoria com a prática, pois quando se fala em ciclo de vida e ciclo reprodutivo, por exemplo, a maioria das explicações é baseada em ilustrações e diagramas, o que torna difícil para esses alunos entenderem.

Esses modelos são apresentados nas mais diversas formas de exploração de texturas, pois o toque passou a ser o significado experimental desses alunos que, além de serem compartilhados com toda a turma, acabam por estabelecer uma conexão direta entre os alunos e o sujeito por meio de texturas e estruturas em diferentes relevos. Dentre os trabalhos que citam a utilização de modelos didáticos para o ensino inclusivo, Cerqueira e Ferreira (1996) apontam que:

Os modelos devem ser criteriosamente escolhidos e, sempre que possível, sua apresentação ao aluno ser acompanhada de explicações verbais objetivas. Objetos muito pequenos podem ser ampliados, para que se tornem perceptíveis detalhes importantes. Objetos situados a grandes distâncias, inacessíveis portanto, precisam ser apresentados sob forma de modelos. O formato de uma nuvem, a forma do sol, da lua, só podem ser apreendidos pelos alunos através de modelos miniaturizados.

Sabendo disso, essas ferramentas são à base de disciplinas como anatomia vegetal, que podem estimular a curiosidade, a imaginação e o interesse dos alunos. As aulas de anatomia vegetal não só apresentam tópicos complexos, mas também apresentam características específicas e simples, exigindo que os professores transmitam os conceitos, trabalhe mais a utilização de exemplos, mas usando recursos dinâmicos, os deficientes visuais e outros colegas podem vivenciar o processo de ensino com mais facilidade, maximizando assim o nível de interatividade da aula.

Podemos concluir que o desenvolvimento cuidadoso do modelo de ensino tátil não apenas ajuda a aprendizagem de pessoas com baixa visão ou cegos, mas também desempenha um papel relacionado na aprendizagem de alunos comuns. A educação inclusiva é um desafio contínuo para todos os profissionais da educação e para a sociedade. É necessário estabelecer uma educação baseada na criatividade, no espírito coletivo e na interdisciplinaridade, e todos esses níveis atenderão os diversos níveis e necessidades dos alunos.

\section{Considerações Finais}

Os modelos didáticos são instrumentos facilitadores de aprendizagens em Botânica, visto que possibilitam uma maior compreensão da morfologia externa, anatomia e fisiologia vegetal, bem como de sistemas complexos, difíceis de serem 
compreendidos sem sua visualização. Podem ser utilizados em diversos níveis de ensino, embora tenha sua utilização com maior frequência seja no Ensino Fundamental. É relevante quanto ao atendimento de alunos portadores de deficiência visual, sendo suporte para a compreensão dos conteúdos da área em questão.

Sua utilização está associada aos objetivos do professor em relação aos conteúdos de ensino e à criatividade do mesmo em relação à sua confecção e manuseio, o qual pode ocorrer em espaços distintos ao da sala de aula. Ao mesmo tempo, encaixa-se em aulas expositivas, aulas práticas, aulas interativas, em exposições, durante intervenções, etc. Podendo ser explorado de diversas maneiras. Sua confecção pode ocorrer com materiais distintos, sendo mais recomendado aqui o biscuit por seu preço acessível e durabilidade.

Estimula-se o uso de modelos didáticos principalmente no estudo da morfologia de plantas angiospérmicas, uma vez que trata-se de um conteúdo mais frequentemente abordado no ensino fundamental, momento de maior curiosidade dos educandos e disponibilidade de tempo para uma abordagem minuciosa de conteúdos. Constatou-se que sua produção e utilização tem expandido no Ensino Superior estimulando a aprendizagem em Botânica e ampliando o acervo de estudo.

Com a revisão sistemática percebe-se a importância da utilização dos modelos didáticos a longa dada, embora em literatura recente não sejam encontrados muitos registros de sua utilização para o ensino de Botânica.

\section{Referências}

Agarez, F. V., Pereira, C., \& Rizzini, C. M. (1994). Botânica: taxonomia, morfologia e reprodução dos angiospermae, chaves para determinação das famílias. (2a ed.), Âmbito Cultural.

Almeida, C. M., Lopes, L. A., \& Lopes, P. T. C. (2015). Sequências didáticas eletrônicas no ensino do corpo humano: comparando o rendimento do ensino tradicional com o ensino utilizando ferramentas tecnológicas. Acta Scientiae, 17(2), 466-482.

Almeida, D. M., Pscheidt, A. C. \& Coelho, C. B. (2019). Inovação em ensino de biologia: o desenvolvimento de uma sequência didática de ensino por investigação utilizando modelos sintéticos de vegetais para as aulas de botânica. INOVAE, 7, 79-93.

Almeida, P. N. (1981). Dinâmica lúdica e jogos pedagógicos para escolares de $1^{o}$ e $2^{o}$ grau. Loyola.

Alves, A. C. (2016). Modelos tridimensionais para ensino de anatomia vegetal para deficientes visuais. In: $57^{\circ}$ Congresso Nacional De Botânica, Vitória, Anais... SSB, 2016.

Anjos, C. C., \& Flores, A. S. (2016). Concepções de estudantes de sétimo ano de uma escola de ensino fundamental sobre forma e função da flor em Boa Vista, Roraima. In: Boletim do museu integrado de Roraima. 10(2), 40-47.

Aoyama, E. M. et. al. (2017). Morfologia floral: modelo didático para o ensino de Botânica. In: $68^{\circ}$ Congresso Nacional De Botânica, Anais... SSB, 2017.

Appezzato-da-Glória, B. \& Carmello-Guerreiro, S. M. (2012). Anatomia vegetal. (3a ed.), Ed. UFV.

Arruda, M. R. P. et.al. (2012). Modelo tridimensional e funcional vegetal: praticidade e dinamismo no ensino de botânica. In: $63^{\circ}$ Congresso Nacional De Botânica, Joinville, Anais...

Barban, J. V. \& Fernandes, H. L. (2010). Zum: desvelando o universo das plantas com arte. <http://www.pg.utfpr.edu.br/sinect/anais2010/ artigos/Ens_Bio/art200.pdf>.

Barros, C. \& Paulinho W. (2009). Ciências: Os seres vivos, (4a ed.), Ártica.

Batista, L. A. B. et. al. (2016). Modelo didático de flores para o ensino de botânica em uma escola pública de Atalaia do Norte-AM. In: $67^{\circ}$ CONGRESSO NACIONAL DE BOTÂNICA, Vitória, Anais... SSB, 2016.

Biagolini, C. H. \& Piacitelli, L. P. (2016). Relato de experiência: o uso da argila na produção de modelos de fitofósseis e seu papel nos processos de ensino e aprendizagem em paleobotânica. Educação Básica Revista, 2(2).

Bizzo, N. (2002). Ciências: fácil ou difícil? (2a ed.), Ática.

Borges, B. T. et. al. (2019). Aulas práticas como estratégias para o ensino de botânica no ensino fundamental. forSCIENCE, 7(2), $231-247$ 10.29069.

Brandão, L. D. \& Carneiro, C. E. (2009). Modelos Dos Ciclos De Vida De Briófitas E Pteridófitas Para Portadores De Deficiência Visual. In: 60 CONGRESSO NACIONAL DE BOTÂNICA, Bahia, Anais... SSB, 2009.

Brasil. (1998). Secretaria da Educação Fundamental. Parâmetros Curriculares Nacionais: ciências naturais: terceiro e quarto ciclos. MEC/SEF.

Canto, E. L. (2012). Ciências Naturais: Aprendendo com o cotidiano, (4a ed.), Moderna. 
Carvalho, D. C. F., Lira, B. S, A. \& Mehlig, Ulf. (2017). Materiais didáticos para o ensino inclusivo de diversidade de plantas com sementes nos cursos de Ciências Biológicas e Naturais. In: $68^{\circ}$ Congresso Nacional De Botânica, Rio de Janeiro, Anais...

Carvalho, I. S. (2011). Paleontologia: Paleovertebrados, paleobotânica. (3a ed.), Interciência.

Ceccantini, G. (2006). Os tecidos vegetais têm três dimensões. Revista Brasileira de Botânica, 29(2), 335-337.

Cerqueira, J. B. \& Ferreira, E. M. B. (1996). Recursos didáticos na educação especial. Revista Benjamin Constant. 5, $24-29$.

Checom, R. L. A. \& Aoyama, E. M. (2014). Anatomia vegetal: modelo didático de botânica para o ensino médio. In: LXV Congresso Nacional De Botânica, Bahia, Anais... SSB, 2014.

Cordeiro, M. R. et. al. (2017). Aulas práticas em anatomia vegetal para deficientes visuais: Proposta de material didático. In: $68^{\circ}$ Congresso Nacional De Botânica, Rio de Janeiro, Anais... SSB, 2017.

Cordeiro, M. R. et. al. (2019). Estratégia didática para ensino inclusivo de alunos com deficiência visual no ensino de anatomia vegetal. In: $70^{\circ}$ Congresso Nacional De Botânica, Maceió, Anais... SSB, 2019.

Costa, P. R. A. M. et. al. (2016). Utilização de porcelana fria na confecção de modelo didático para o ensino de anatomia vegetal. In: III Congresso Nacional De Educação, Natal, Anais... CONEDU, 2016.

Custódio, L. N., Faria, R. L. \& Carmo-Oliveira, R. (2007). Da flor ao fruto: um modelo para o ensino de botânica. In: $58^{\circ}$ CONGRESSO NACIONAL DE BOTÂNICA, São Paulo, Anais... SSB, 2007.

Duckworth, E. (1994). Cómo tener ideas maravillosas y otros ensayos sobre cómo enseñar y aprender. Visor.

Fonseca, L. (2000). Ensino de ciências e saber popular. Saúde e Educação. DP\&A.

Fontes, G. S. \& Aoyama, E. M. (2016). Modelo didático de fruto para o ensino aprendizagem de botânica. In: $67^{\circ}$ Congresso Nacional De Botânica, Vitória, Anais... SSB, 2016.

Fontes, G. S., Elias, L. \& Aoyama, E. M. (2019). Flora nativa no ensino de botânica: proposta de modelo didático de fruto. Experiências em Ensino de Ciências. 14(2), 385-394.

Garcia, M. F. F. (2000). Repensando a Botânica. In: COLETÂNEA DO $7^{\circ}$ ENCONTRO PERSPECTIVAS DO ENSINO DE BIOLOGIA.

Judd, W. S. et.al. (2009). Sistemática Vegetal, Um Enfoque Filogenético. $3^{\mathrm{a}}$ ed. Porto Alegre - RS Armed editora S.A.

Junior-Silva, W., Cabrini, E. C. (2018). Modelos didáticos como ferramenta de ensino nas aulas de botânica no ensino superior. In: $69^{\circ}$ Congresso Nacional De Botânica, Cuiabá, Anais... SSB, 2018.

Justina, D. et.al. (2003). Modelos didáticos no ensino de Genética. In: Seminário De Extensão Da Unioeste, 3. Cascavel. Anais... p.135-40.

Justina, L. A. D. \& Ferla, M. R. (2005). A utilização de modelos didáticos no ensino de genética - exemplo de representação de compactação do DNA eucarioto. Arquivos do Museu Dinâmico Interdisciplinar, 10(2), 35-40.

Kerbauy, G.B. (2012). Fisiologia Vegetal. (2a ed.), Guanabara Koogan.

Krasilchik, M. (1996). Prática de Biologia. (6a ed.), Habra. P.67.

Leme, F. M. et. al. (2018). Ensino de botânica e educação ambiental: modelos didáticos e oficinas pedagógicas. Revista eletrônica Educação Ambiental em Ação. http://www.revistaea.org/pf.php?idartigo=2122.

Lima, I. G., Jesus, S. C., \& Santos S. P. (2012). O uso de jogo didático e modelos como recursos para o ensino de botânica. In: $63^{\circ}$ Congresso Nacional De Botânica, Joinville, Anais... SSB, 2012.

Marinho, B, F. et. al. (2018). Construção de um modelo didático para o ensino de botânica na disciplina de práticas de ensino em biologia. In: V CONGRESSO NACIONAL DE EDUCAÇÃO, Olinda, Anais... CONEDU, 2018.

Martin, R. E. (1999). Taphonomy: a process approach. Reino Unido: Cambridge. (Cambrige Paleobiology Serie, 4).

Matos, C. H. C. et.al. (2009). Utilização de modelos didáticos no ensino de entomologia. Revista de biologia e ciências da terra, v.9, n1, p 1-5. Disponível em: <http://joaootavio.com.br/bioterra/workspace/uploads/artigos/3matos51816c32b2719.pdf> Acessado em: 19 de Junho 2018.

Mendoza, A. Y.G. et. al. (2017). A construção do conhecimento de plantas carnívoras pelos alunos do ensino fundamental no município de Benjamin Constant-AM. In: $68^{\circ}$ Congresso Nacional De Botânica, Rio de Janeiro, Anais... SSB, 2017.

Nabors, M. W. Introdução a Botânica. Ed. Rocha LTDA, 2012.

Nariane, Q. V. et.al. (2010) Modelos Didático-pedagógicos: Estratégias Inovadoras para o Ensino de Biologia. In: SIMPÓSIO NACIONAL DE NSINO DE CIÊNCIAS E TECNOLOGIA. Belém, Pará. Anais... UEPA: p.1-13.

Pereira, F. L. (2012). Efeitos do exercício físico contra-resistência em idosos saudáveis: revisão sistemática. Monografia. (Graduação Bacharel em Educação Física) - Centro de desportos (CDS), Departamento de educação física (DEF), Universidade Federal de Santa Catarina. Florianópolis.

Perini1, M. \& Rossini, J. (2018). Aplicação de modelos didáticos no ensino de biologia floral. International Scientific Journal, 3(13), 58-71. 
Pesamosca, A. M. et. al. (2012). Modelos didáticos: uma alternativa metodológica no ensino de botânica. In: $63^{\circ}$ Congresso Nacional De Botânica, Joinville, Anais... SSB, 2012.

Ramos, M. L. \& Lima, R. A.. (2019). Modelos didáticos para o ensino de Botânica no ensino superior. In: $70^{\circ}$ Congresso Nacional De Botânica, Maceió, Anais... SSB, 2019.

Rauber, I. M. F. et.al. (2006) A importância da utilização de modelos didáticos do ciclo de vida do Ascomycota para o ensino de botânica. In: $57^{\circ}$ Congresso Nacional De Botânica, Gramado, Anais... SSB, 2006.

Raven, P. H., Evert, R. F., Eichhorn, S. E. (2007) Biologia Vegetal. (7a ed.), Guanabara Koogan.

Ribeiro, J. M. M. \& Carvalho, M. A. S. (2017). Utilização de modelos didáticos no ensino de botânica e suas implicações no processo de ensino e aprendizagem. Revista Sapiência: sociedade, saberes e práticas educacionais, 6(1), 17-37.

Rocha, L. et. al. (2018) Um modelo didático da flor para o ensino de sua morfologia e dos processos associados à reprodução das angiospermas. In: $69^{\circ}$ CONGRESSO NACIONAL DE BOTÂNICA, Cuiabá, Anais... Cuiabá: SSB, 2018.

Rother, J. P. S. F. S. R. (2014) Modelos Anatômicos como recurso didático em aulas práticas de Ciências e Biologia. Monografia (Graduação em Licenciatura em Ciências Biológicas) - Setor de Educação, Departamento de Teoria e Prática de Ensino, Universidade Federal do Paraná. Curitiba.

Sales, G. N. B. \& Lima, J. R.(2014). Uso de modelos didáticos em aulas de citologia e histologia vegetal: estudo de caso em uma turma de agronomia. In: LXV Congresso Nacional De Botânica. Bahia, Anais... SSB, 2014.

Saraiva, D. P. et. al. (2018). Da folha carpelar ao fruto: uso de modelos de biscuit no ensino de morfologia vegetal. In: $69^{\circ}$ CONGRESSO NACIONAL DE BOTÂNICA, 2018, Cuiabá, Anais... SSB, 2018.

Silva, A. A., Filha-Silva, R. T. \& Freitas, S. R. S. (2016) Utilização de modelo didático como metodologia complementar ao ensino de anatomia celular. Biota Amazônia. 6(3), 17-21.

Silva, H. C. et.al. (2009) A abordagem de atividades práticas no ensino da botânica nos livros didáticos de biologia do ensino médio.: <http://www.eventosufrpe.com.br/jepeX2009/cd/resumos/R0610-1.pdf>.

Silva, M, S. et. al. (2019). Elaboração de modelo didático táctil de plantas avasculares no ensino de botânica para portadores de baixa visão. In: VI Congresso Nacional De Educação, Fortaleza, Anais... CONEDU.

Silva, P. H. et.al. (2017) Modelos didáticos como instrumento facilitador em aulas de botânica. VII EREBIO. Encontro Regional de Ensino de Biologia, Regional 5, Nordeste. Universidade Regional do Cariri - URCA, 06 a 09 de setembro de 2017.

Souza, D. C., Andrade, G. L. P. \& Nascimento, J. A. F.(2008) Produção de Material Didático-Pedagógico Alternativo para o Ensino do Conceito Pirâmide Ecológica: Um Subsídio a Educação Cientifica e Ambiental. In: Fórum Ambiental da Alta Paulista. São Paulo, São Paulo. Anais... São Paulo: ANAP. p.97130.

Souza, V. C., Flores, T. B. \& Lorenzi, H. (2013) Introdução à Botânica: morfologia. São Paulo: Instituto Plantarum de Estudos da Flora.

Traverse, A. (1998) Paleopalynology. Dederecht, Springer, (2 ed,), 814.

Ventrella, M.C. Anatoblocos: blocos didáticos para o estudo da anatomia vegetal. UFV, Cead, 2016. 83p. https://www2.cead.ufv.br/serieconhecimento/wpcontent/uploads/2016/07/AnatoblocosFinal_04072016.pdf.

Vidal, W. N. \& Vidal, M. R. R. (2000) Botânica - organografia, quadros sinóticos ilustrados de fanerógamos. (4a ed.), UFV.

Ward, L. F. (1885). Sketch of paleobotany. US Geological Survey Annual Report, 5, 357-452.

Wilsek, M. A. G. \& Tosin, J. A. P.(2009). Ensinar e Aprender Ciências no Ensino Fundamental com Atividades Investigativas através da Resolução de Problemas. http://www.diaadiaeducacao.pr.gov.br/portals/pde/arquivos/1686-8.pdf. 